\title{
Analyticity of the entropy for some random walks
}

\author{
François Ledrappier
}

\begin{abstract}
We consider non-degenerate, finitely supported random walks on a free group. We show that the entropy and the linear drift vary analytically with the probability of constant support.
\end{abstract}

Mathematics Subject Classification (2010). 60G50, 60B15.

Keywords. Entropy, free group.

\section{Introduction}

Let $F$ be a finitely generated group and for $x \in F$, denote $|x|$ the word length of $x$. Let $p$ be a finitely supported probability measure on $F$ and define inductively, with $p^{(0)}$ being the Dirac measure at the identity $e$,

$$
p^{(n)}(x)=\left[p^{(n-1)} \star p\right](x)=\sum_{y \in F} p^{(n-1)}\left(x y^{-1}\right) p(y) .
$$

Some of the asymptotic properties of the probabilities $p^{(n)}$ as $n \rightarrow \infty$ are reflected in two non-negative numbers, the entropy $h_{p}$ and the linear drift $\ell_{p}$ :

$$
h_{p}:=\lim _{n}-\frac{1}{n} \sum_{x \in F} p^{(n)}(x) \ln p^{(n)}(x), \quad \ell_{p}:=\lim _{n} \frac{1}{n} \sum_{x \in F}|x| p^{(n)}(x) .
$$

Erschler asks whether $h_{p}$ and $\ell_{p}$ depend continuously on $p$ ([Er]). In this note, we fix a finite set $B \subset F$ such that $\bigcup_{n} B^{n}=F$ and we consider probability measures in $\mathcal{P}(B)$, where $\mathcal{P}(B)$ is the set of probability measures $p$ such that $p(x)>0$ if, and only if, $x \in B$. The set $\mathcal{P}(B)$ is naturally identified with an open subset of the probabilities on $B$ which is an open bounded convex domain in $\mathbb{R}^{|B|-1}$. We show:

Theorem 1.1. Assume that $F=\mathbb{F}_{d}$ is the free group with $d$ generators, $B$ is a finite subset of $F$ such that $\bigcup_{n} B^{n}=F$. Then, with the above notation, the functions $p \mapsto h_{p}$ and $p \mapsto \ell_{p}$ are real analytic on $\mathcal{P}(B)$. 
Continuity of the entropy and of the linear drift is known for probabilities with first moment on a Gromov-hyperbolic group ([EK]). Also in the case when $B$ is a set of free generators, there are formulas for the entropy and the linear drift which show that they are real analytic functions of the directing probability (see [De2] or imbed $[D M]$ in the formulas (1) and (2) below). Similar formulas have been found for braid groups ([M]) and free products of finite groups or graphs ([MM], [G1], [G2]), but as soon as the set $B$ is not reduced to the natural generating set, there is no direct formula for $h_{p}$ or $\ell_{p}$ in terms of $p$.

The ratio $h_{p} / \ell_{p}$ has a geometric interpretation as the Hausdorff dimension $D_{p}$ of the unique stationary measure for the action of $F$ on the space $\partial F$ of infinite reduced words. It follows from Theorem 1.1 that this dimension $D_{p}$ is also real analytic in $p$, see Corollary 2.1 below for a more precise statement. Ruelle ([R3]) proved that the Hausdorff dimension of the Julia set of a rational function, as long as it is hyperbolic, depends real analytically of the parameters and our approach is inspired by [R3]. We first review properties of the random walk on $F$ directed by a probability $p$. In particular, we can express $h_{p}$ and $\ell_{p}$ in terms of the exit measure $p^{\infty}$ of the random walk on the boundary $\partial F$ (see [Le] and Section 2 for background and notation). We then express this exit measure using thermodynamical formalism: if one views $\partial F$ as a one-sided subshift of finite type, the exit measure $p^{\infty}$ is the isolated eigenvector of maximal eigenvalue for a dual transfer operator $\mathscr{L}_{p}^{*}$ involving the Martin kernel of the random walk. Finally, from the description of the Martin kernel by Derriennic ([De1]), we prove that the mapping $p \mapsto \mathscr{L}_{p}$ is real analytic. The proof uses contractions in projective metric on complex cones ([Ru], [Du1]), and I want to thank Loïc Dubois for useful comments. Regularity of $p \mapsto p^{\infty}$ and Theorem 1.1 follow.

Our argument may apply to other similar settings. For instance, let $\pi: \mathbb{F}_{d} \rightarrow$ $\mathrm{SO}(k, 1)$ be a faithful Schottky representation of the free group $\mathbb{F}_{d}$ as a convex cocompact group of $\mathrm{SO}(k, 1)$. Namely, $\mathrm{SO}(k, 1)$ is considered as a group of isometries of the hyperbolic space $\mathbb{H}^{k}$ and there are $2 d$ disjoint open halfspaces $H_{a}$ associated to the generators and their inverses in such a way that $\pi(a)$ sends the complement of $H_{a^{-1}}$ onto the closure of $H_{a}$ in $\mathbb{H}^{k}$. Then another natural asymptotic quantity is the Lyapunov exponent

$$
\gamma_{p}:=\lim _{n} \frac{1}{n} \sum_{x \in F} p^{(n)}(x) \ln \|\pi(x)\|,
$$

where $\|\cdot\|$ is some norm on matrices.

Theorem 1.2. Assume that $\mathbb{F}_{d}$ is represented as a convex cocompact subgroup of $\mathrm{SO}(k, 1)$ as above, and $B$ is a finite subset $B \subset F$ such that $\bigcup_{n} B^{n}=F$. Then the function $p \mapsto \gamma_{p}$ is a real analytic function on $\mathcal{P}(B)$.

Analyticity of the exponent of an independent random product of matrices is known for positive matrices $([\mathrm{R} 2],[\mathrm{P}],[\mathrm{H}])$. Here we show it for matrices in some 
discrete subgroup. It is possible that our approach yield similar results for more general discrete subgroups of $\mathrm{SO}(k, 1)$ or even for all Gromov-hyperbolic groups.

In the note, the letter $C$ stands for a real number independent of the other variables, but which may vary from line to line. In the same way, the letter $\mathcal{O}_{p}$ stands for a neighborhood of $p \in \mathcal{P}(B)$ in $\mathbb{C}^{B}$ which may vary from line to line.

\section{Convolutions of $p$}

We recall in this section the properties of the convolutions $p^{(n)}$ of a finitely supported probability measure $p$ on the free group $\mathbb{F}_{d}=F$. We follow the notation from [Le]. Any element of $F$ has a unique reduced word representation in generators $\left\{a_{1}, \ldots, a_{d}, a_{-1}, \ldots, a_{-d}\right\}$. Set $\delta(x, x)=0$ and, for $x \neq x^{\prime}, \delta\left(x, x^{\prime}\right)=\exp -(x \wedge$ $\left.x^{\prime}\right)$, where $\left(x \wedge x^{\prime}\right)$ is the number of common letters at the beginning of the reduced word representations of $x$ and $x^{\prime}$. Then $\delta$ defines a metric on $F$ and extends to the completion $F \cup \partial F$ with respect to $\delta$. The boundary $\partial F$ is a compact space which can be represented as the space of infinite reduced words. Then the distance between two distinct infinite reduced words $\xi$ and $\xi^{\prime}$ is given by

$$
\delta\left(\xi, \xi^{\prime}\right)=\exp -\left(\xi \wedge \xi^{\prime}\right),
$$

where $\left(\xi \wedge \xi^{\prime}\right)$ is the length of the initial common part of $\xi$ and $\xi^{\prime}$.

There is a natural continuous action of $F$ over $\partial F$ which extends the left action of $F$ on itself: one concatenates the reduced word representation of $x \in F$ at the beginning of the infinite word $\xi$ and one obtains a reduced word by making the necessary reductions. A probability measure $\mu$ on $\partial F$ is called stationary if it satisfies

$$
\mu=\sum_{x \in F} p(x) x_{*} \mu .
$$

There is a unique stationary probability measure on $\partial F$, denoted by $p^{\infty}$, and the entropy $h_{p}$ and the linear drift $\ell_{p}$ are given by

$$
\begin{aligned}
& h_{p}=-\sum_{x \in F}\left(\int_{\partial F} \ln \frac{d x_{*}^{-1} p^{\infty}}{d p^{\infty}}(\xi) d p^{\infty}(\xi)\right) p(x), \\
& \ell_{p}=\sum_{x \in F}\left(\int_{\partial F} \theta_{\xi}\left(x^{-1}\right) d p^{\infty}(\xi)\right) p(x),
\end{aligned}
$$

where $\theta_{\xi}(x)=|x|-2(\xi \wedge x)=\lim _{y \rightarrow \xi}\left(\left|x^{-1} y\right|-|y|\right)$ is the Busemann function.

Observe that in both expressions, the sum is a finite sum over $x \in B$. In the case of a finitely supported random walk on a general group, formula (1) holds, but with $\left(\partial F, p^{\infty}\right)$ replaced by the Poisson boundary of the random walk (see [Fu], [Ka]); formula (2) also holds, but with $\left(\partial F, p^{\infty}\right)$ replaced by some stationary measure on the Busemann boundary of the group ([KL]). 
Recall that in the case of the free group the Hausdorff dimension of the measure $p^{\infty}$ on $(\partial F, \delta)$ is given by $h_{p} / \ell_{p}$ ([Le], Theorem 4.15). So we have the following corollary of Theorem 1.1:

Corollary 2.1. Assume that $F=\mathbb{F}_{d}$ is the free group with $d$ generators, $B$ is a finite subset of $F$ such that $\bigcup_{n} B^{n}=F$. Then, with the above notation, the Hausdorff dimension of the stationary measure on $(\partial F, \delta)$ is a real analytic function of $p$ in $\mathcal{P}(B)$.

The Green function $G(x)$ associated to $(F, p)$ is defined by

$$
G(x)=\sum_{n=0}^{\infty} p^{(n)}(x)
$$

(see Proposition 3.2 below for the convergence of the series). For $y \in F$, the Martin kernel $K_{y}$ is defined by

$$
K_{y}(x)=\frac{G\left(x^{-1} y\right)}{G(y)} .
$$

Derriennic ([De1]) showed that $y_{n} \rightarrow \xi \in \partial F$ if, and only if, the Martin kernels $K_{y_{n}}$ converge towards a function $K_{\xi}$, called the Martin kernel at $\xi$. We have (see e.g. [Le] (3.11)):

$$
\frac{d x_{*} p^{\infty}}{d p^{\infty}}(\xi)=K_{\xi}(x)
$$

\section{Random walk on $F$}

The quantities introduced in Section 2 can be associated with the trajectories of a random walk on $F$. In this section, we recall the corresponding notation and properties. Let $\Omega=F^{\mathbb{N}}$ be the space of sequences of elements of $F, M$ the product probability $p^{\mathbb{N}}$. The random walk is described by the probability $\mathbb{P}$ on the space of paths $\Omega$, the image of $M$ by the mapping

$$
\left(\omega_{n}\right)_{n \in \mathbb{Z}} \mapsto\left(X_{n}\right)_{n \geq 0},
$$

where $X_{0}=e$ and $X_{n}=X_{n-1} \omega_{n}$ for $n>0$. In particular, the distribution of $X_{n}$ is the convolution $p^{(n)}$. The notation $p^{\infty}$ reflects the following.

Theorem 3.1 (Furstenberg, [Le], Theorem 1.12). There is a mapping $X_{\infty}: \Omega \rightarrow \partial F$ such that

$$
\lim _{n} X_{n}(\omega)=X_{\infty}(\omega)
$$

for $M$-a.e. $\omega$. The image measure $p^{\infty}$ is the only stationary probability measure on $\partial F$. 
For $x, y \in F$, let $u(x, y)$ be the probability of eventually reaching $y$ when starting from $x$. By left invariance, $u(x, y)=u\left(e, x^{-1} y\right)$. Moreover, by the strong Markov property, $G(x)=u(e, x) G(e)$ so that we have

$$
K_{y}(x)=\frac{u(x, y)}{u(e, y)} .
$$

By definition, we have $0<u(x, y) \leq 1$. The number $u(x, y)$ is given by the sum of the probabilities of the paths going from $x$ to $y$ which do not visit $y$ before arriving at $y$.

Proposition 3.2. Let $p \in \mathcal{P}(B)$. There are numbers $C$ and $\zeta, 0<\zeta<1$, and $a$ neighborhood $\mathcal{O}_{p}$ of $p$ in $\mathbb{C}^{B}$ such that for all $q \in \mathcal{O}_{p}$, all $x \in F$ and all $n \geq 0$,

$$
|q|^{(n)}(x) \leq C \zeta^{n} .
$$

Proof. Let $q \in \mathbb{C}^{B}$. Consider the convolution operator $P_{q}$ in $\ell_{2}(F, \mathbb{R})$ defined by

$$
P_{q} f(x)=\sum_{y \in F} f\left(x y^{-1}\right)|q|(y) .
$$

Derriennic and Guivarc'h ([DG]) showed that, for $p \in \mathcal{P}(B), P_{p}$ has spectral radius smaller than one. In particular, there exists $n_{0}$ such that the operator norm of $P_{p}^{n_{0}}$ in $\ell_{2}(F)$ is smaller than one. Since $B$ and $B^{n_{0}}$ are finite, there is a neighborhood $\mathcal{O}_{p}$ of $p$ in $\mathbb{C}^{B}$ such that for all $q \in \mathcal{O}_{p},\left\|P_{q}^{n_{0}}\right\|_{2}<\lambda$ for some $\lambda<1$ and $\left\|P_{q}^{k}\right\|_{2} \leq C$ for $1 \leq k \leq n_{0}$. It follows that for all $q \in \mathcal{O}_{p}$, all $n \geq 0$,

$$
\left\|P_{q}^{n}\right\|_{2} \leq C \lambda^{\left[n / n_{0}\right]} .
$$

In particular, $|q|^{(n)}(x)=\left|\left[P_{q}^{n} \delta_{e}\right](x)\right| \leq\left|P_{q}^{n} \delta_{e}\right|_{2} \leq C \lambda^{\left[n / n_{0}\right]}\left|\delta_{e}\right|_{2} \leq C \lambda^{\left[n / n_{0}\right]}$ for all $x \in F$.

Fix $p \in \mathcal{P}(B)$. For $x \in F, V$ a finite subset of $F$, and $v \in V$, let $\alpha_{x}^{V}(v)$ be the probability that the first visit in $V$ of the random walk starting from $x$ occurs at $v$. We have $0<\sum_{v \in V} \alpha_{x}^{V}(v) \leq 1$ and

Proposition 3.3. Fix $x, V$, and $v$. The mapping $p \mapsto \alpha_{x}^{V}(v)$ extends to an analytic function on a neighborhood of $\mathcal{P}(B)$ in $\mathbb{C}^{B}$.

Proof. The number $\alpha_{x}^{V}(v)$ can be written as the sum of the probabilities $\alpha_{x}^{n, V}(v)$ of entering $V$ at $v$ in exactly $n$ steps. The function $p \mapsto \alpha_{x}^{n, V}(v)$ is a polynomial of degree $n$ on $\mathcal{P}(B)$ :

$$
\alpha_{x}^{n, V}(v)=\sum_{\varepsilon} q_{i_{1}} q_{i_{2}} \ldots q_{i_{n}}
$$


where $\mathcal{E}$ is the set of paths $\left\{x, x i_{1}, x i_{1} i_{2}, \ldots, x i_{1} i_{2} \ldots i_{n}=v\right\}$ of length $n$ made of steps in $B$ which start from $x$ and enter $V$ in $v$. By Proposition 3.2, there is a neighbourhood $\mathcal{O}_{p}$ of $p$ in $\mathcal{P}(B)$ and numbers $C, \zeta, 0<\zeta<1$, such that for $q \in \mathcal{O}_{p}$ and for all $y \in F$,

$$
|q|^{(n)}(y) \leq C \xi^{n} .
$$

It follows that for $q \in \mathcal{O}_{p}$,

$$
\left|\alpha_{x}^{n, V}(v)\right| \leq C|q|^{(n)}\left(x^{-1} v\right) \leq C \zeta^{n} .
$$

Therefore, $q \mapsto \alpha_{x}^{V}(v)$ is given locally by a uniformly converging series of polynomials, it is an analytic function on $\mathcal{O}:=\bigcup_{p} \mathcal{O}_{p}$.

\section{Barriers and Hölder property of the Martin kernel}

Set $r=\max \{|x| \mid x \in B\}$. A set $V$ is called a barrier between $x$ and $y$ if $\delta(x, y)>r$ and if there exist two points $z$ and $z^{\prime}$ of the geodesic between $x$ and $y$, distinct from $x$ and $y$ such that $\delta\left(z, z^{\prime}\right)=r-1$ and $V$ is the intersection of the two balls of radius $r-1$ centered at $z$ and at $z^{\prime}$. The basic geometric lemma is the following:

Lemma 4.1 ([De1], Lemme 1). If $x$ and $y$ admit a barrier $V$, then every trajectory of the random walk starting from $x$ and reaching $y$ has to visit $V$ before arriving at $y$.

For $V, W$ finite subsets of $F$, denote by $A_{V}^{W}$ the matrix such that the row vectors are the $\alpha_{v}^{W}(w), w \in W$. In particular, if $W=\{y\}$, set $u_{V}^{y}$ equal to the (column) vector

$$
u_{V}^{y}=A_{V}^{\{y\}}=\left(\alpha_{v}^{\{y\}}(y)\right)_{v \in V}=(u(v, y))_{v \in V} .
$$

With this notation, Lemma 4.1 and the strong Markov property yield that if $x$ and $y$ admit $V$ as a barrier, then

$$
u(x, y)=\sum \alpha_{x}^{V}(v) u(v, y)=\left\langle\alpha_{x}^{V}, u_{V}^{y}\right\rangle,
$$

with the natural scalar product on $\mathbb{R}^{V}$. Then Derriennic makes two observations: first, this formula iterates when one has $k$ successive disjoint barriers between $x$ and $y$, and secondly there are only a finite number of possible matrices $A_{V}^{W}$ when $V$ and $W$ are successive disjoint barriers with $\delta(V, W)=1$. This gives the following formula for $u(x, y)$ :

Lemma 4.2 ([De1], Lemme 2). Let $p \in \mathcal{P}(B)$. There are $N$ square matrices with the same dimension $A_{1}, \ldots, A_{N}$, depending on $p$, such that for any $x, y \in$ $F$ : if $V_{1}, V_{2}, \ldots, V_{k}$ are disjoint successive barriers between $x$ and $y$ such that 
$\delta\left(V_{i}, V_{i+1}\right)=1$ for $i=1, \ldots, k-1$, then there are $(k-1)$ indices $j_{1}, \ldots j_{k-1}$, depending only on the sequence $V_{i}$, such that

$$
u(x, y)=\left\langle\alpha_{x}^{V_{1}}, A_{j_{1}} \ldots A_{j_{k-1}} u_{V_{k}}^{y}\right\rangle .
$$

By construction, the matrices $A_{j}$ have non-negative entries and $\sum_{w} A_{j}(v, w) \leq$ 1. Moreover, we have the following properties:

Proposition 4.3 ([De1], Corollaire 1). Assume that the set $B$ contains the generators and their inverses. Then for each $p \in \mathcal{P}(B)$, for each $j=1, \ldots, N$, the matrix $A_{j}$ has all its 0 entries in full columns.

From the proof of Proposition 4.3, if the set $B$ contains the generators and their inverses and $A_{j}=A_{V_{j}}^{V_{j+1}}$, columns of 0's correspond to the subset $W_{j+1}$ of points in $V_{j+1}$ which cannot be entry points from paths starting in $V_{j}$. In particular, they depend only of the geometry of $B$ and are the same for all $p \in \mathcal{P}(B)$.

We may - and we shall from now on - assume that the set $B$ contains the generators and their inverses. Indeed, since $h_{p^{(k)}}=k h_{p}$ and $\ell_{p^{(k)}}=k \ell_{p}$, we can replace in Theorem 1.1 the probability $p$ by a convolution of order high enough that the generators and their inverses have positive probability. Then, by Proposition 4.3, the matrices $A_{j}(q)$ have the same columns of zeros for all $q \in \mathcal{P}(B)$.

Proposition 4.4. For each $j=1, \ldots, N$, the mapping $p \mapsto A_{j}$ extends to an analytic function on a neighborhood of $\mathcal{P}(B)$ in $\mathbb{C}^{B}$ into the set of complex matrices with the same configuration of zeros as $A_{j}$.

Proof. The proof is completely analogous to the proof of Proposition 3.3; one may have to take a smaller neighborhood for the sake of avoiding introducing new zeros.

We are interested in the function $\Phi: \partial F \rightarrow \mathbb{R}, \Phi(\xi)=-\ln K_{\xi}\left(\xi_{1}\right)$. By (3), (4) and Deriennic's theorem, we have

$$
\begin{aligned}
\Phi(\xi) & =-\ln \lim _{n \rightarrow \infty} K_{\xi_{1} \xi_{2} \ldots \xi_{n}}\left(\xi_{1}\right) \\
& =-\ln \lim _{n \rightarrow \infty} \frac{u\left(\xi_{1}, \xi_{1} \xi_{2} \ldots \xi_{n}\right)}{u\left(e, \xi_{1} \xi_{2} \ldots \xi_{n}\right)} \\
& =-\ln \lim _{k \rightarrow \infty} \frac{\left\langle\alpha_{\xi_{1}}^{V_{1}(\xi)}, A_{j_{1}}(\xi) \ldots A_{j_{k-1}}(\xi) u_{V_{k}(\xi)}^{y_{k}}\right\rangle}{\left\langle\alpha_{e}^{V_{1}(\xi)}, A_{j_{1}}(\xi) \ldots A_{j_{k-1}}(\xi) u_{V_{k}(\xi)}^{y_{k}}\right\rangle},
\end{aligned}
$$

where $A_{j_{s}}(\xi)=A_{V_{s}(\xi)}^{V_{s+1}(\xi)}$, the $V_{s}(\xi)$ are successive disjoint barriers between $\xi_{1}$ and $\xi$ with $\delta\left(V_{s}(\xi), V_{s+1}(\xi)\right)=1$ for all $s>1, \delta\left(\xi_{1}, V_{1}\right)=1$, and $y_{k}$ is the closest point beyond $V_{k}$ on the geodesic from $\xi_{1}$ to $\xi$. 
Define on the non-negative convex cone $C_{0}$ in $\mathbb{R}^{m}$ the projective distance between half lines as

$$
\vartheta(f, g):=\left|\ln \left[f, g, h, h^{\prime}\right]\right|,
$$

where $h, h^{\prime}$ are the intersections of the boundaries of the cone with the plane $(f, g)$ and $\left[f, g, h, h^{\prime}\right]$ is the cross ratio of the four directions in the same plane. Represent the space of directions as the sector of the unit sphere $D=C_{0} \cup S^{m-1}$; then $\vartheta$ defines a distance on $D$. Let $A$ be a $m \times m$ matrix with non-positive entries, and let $T: D \rightarrow D$ be the projective action of $A$. Then, by [Bi],

$$
\vartheta(T f, T g) \leq \beta \vartheta(f, g), \quad \text { where } \beta=\tanh \left(\frac{1}{4} \operatorname{Diam} T(D)\right) .
$$

When $A_{j}$ is one of the matrices of Lemma 4.2, it acts on $\mathbb{R}^{V}$ and the image $T_{j}(D)$ has finite diameter so that $\beta_{j}:=\tanh \left(\frac{1}{4} \operatorname{Diam} T_{j}(D)\right)<1$. Set $\beta_{0}:=\max _{j=1, \ldots, N} \beta_{j}$. Then $\beta_{0}<1$.

Set $f_{k}(\xi):=\frac{u_{V_{k}(\xi)}^{y_{k}}}{\left\|u_{V_{k}(\xi)}^{y_{k}}\right\|}, \alpha(\xi):=\alpha_{e}^{V_{1}(\xi)}, \alpha_{1}(\xi):=\alpha_{\xi_{1}}^{V_{1}(\xi)}$. For all $\xi, f_{k}(\xi) \in D$ and $\alpha(\xi), \alpha_{1}(\xi) \in C_{0}-\{0\}$. The above formula for $\Phi(\xi)$ becomes

$$
\Phi(\xi)=-\ln \lim _{k \rightarrow \infty} \frac{\left\langle\alpha_{1}(\xi), T_{j_{1}}(\xi) \ldots T_{j_{k-1}}(\xi) f_{k}(\xi)\right\rangle}{\left\langle\alpha(\xi), T_{j_{1}}(\xi) \ldots T_{j_{k-1}}(\xi) f_{k}(\xi)\right\rangle}
$$

Proposition 4.5. Fix $p \in \mathcal{P}$. The function $\xi \mapsto \Phi(\xi)$ is Hölder continuous on $\partial F$.

Proof. Let $\xi$, $\xi^{\prime}$ be two points of $\partial F$ with $\delta\left(\xi, \xi^{\prime}\right) \leq \exp (-((n+1) r+1))$. The points $\xi$ and $\xi^{\prime}$ have the same first $(n+1) r+1$ coordinates. In particular, $V_{s}(\xi)=V_{s}\left(\xi^{\prime}\right)$ for $1 \leq s \leq n$. By using (6), we see that $\Phi\left(\xi^{\prime}\right)-\Phi(\xi)$ is given by the limit, as $k$ goes to infinity, of

$$
\ln \frac{\left\langle\alpha_{1}(\xi), T_{j_{1}}(\xi) \ldots T_{j_{k-1}}(\xi) f_{k}(\xi)\right\rangle}{\left\langle\alpha_{1}\left(\xi^{\prime}\right), T_{j_{1}}\left(\xi^{\prime}\right) \ldots T_{j_{k-1}}\left(\xi^{\prime}\right) f_{k}\left(\xi^{\prime}\right)\right\rangle} \frac{\left\langle\alpha\left(\xi^{\prime}\right), T_{j_{1}}\left(\xi^{\prime}\right) \ldots T_{j_{k-1}}\left(\xi^{\prime}\right) f_{k}\left(\xi^{\prime}\right)\right\rangle}{\left\langle\alpha(\xi), T_{j_{1}}(\xi) \ldots T_{j_{k-1}}(\xi) f_{k}(\xi)\right\rangle} .
$$

We have $\alpha_{1}(\xi)=\alpha_{1}\left(\xi^{\prime}\right)=: \alpha_{1}, \alpha(\xi)=\alpha\left(\xi^{\prime}\right)=: \alpha$ and $T_{j_{s}}(\xi)=T_{j_{s}}\left(\xi^{\prime}\right)=: T_{j_{s}}$ for $s=1, \ldots, n$. Moreover, for any $f, f^{\prime} \in D$,

$$
\begin{gathered}
\vartheta\left(T_{j_{1}}(\xi) \ldots T_{j_{k-1}}(\xi) f, T_{j_{1}}\left(\xi^{\prime}\right) \ldots T_{j_{k-1}}\left(\xi^{\prime}\right) f^{\prime}\right) \\
=\vartheta\left(T_{j_{1}} \ldots T_{j_{n-1}} g_{k}, T_{j_{1}} \ldots T_{j_{n-1}} g_{k}^{\prime}\right)
\end{gathered}
$$

for $g_{k}=T_{j_{n}} T_{j_{n+1}}(\xi) \ldots T_{j_{k-1}}(\xi) f, g_{k}^{\prime}=T_{j_{n}} T_{j_{n+1}}\left(\xi^{\prime}\right) \ldots T_{j_{k-1}}\left(\xi^{\prime}\right) f^{\prime}$.

We have $\vartheta\left(g_{k}, g_{k}^{\prime}\right) \leq$ Diam $T_{j_{n}} D<\infty$ and, by repeated application of (5),

$$
\vartheta\left(T_{j_{1}} \ldots T_{j_{n-1}} g_{k}, T_{j_{1}} \ldots T_{j_{n-1}} g_{k}^{\prime}\right) \leq \beta_{0}^{n-1} \vartheta\left(g_{k}, g_{k}^{\prime}\right) \leq C \beta_{0}^{n}
$$

Using all the above notation, we get

$$
\Phi(\xi)-\Phi\left(\xi^{\prime}\right)=\ln \lim _{k} \frac{\left\langle\alpha_{1}, T_{j_{1}} \ldots T_{j_{n-1}} g_{k}^{\prime}\right\rangle}{\left\langle\alpha_{1}, T_{j_{1}} \ldots T_{j_{n-1}} g_{k}\right\rangle} \frac{\left\langle\alpha, T_{j_{1}} \ldots T_{j_{n-1}} g_{k}\right\rangle}{\left\langle\alpha, T_{j_{1}} \ldots T_{j_{n-1}} g_{k}^{\prime}\right\rangle} .
$$


As $\xi$ varies, $\alpha$ and $\alpha_{1}$ belong to a finite family of vectors of $C_{0}-\{0\}$. It then follows from (7) that $\left|\Phi(\xi)-\Phi\left(\xi^{\prime}\right)\right| \leq C \beta_{0}^{n}$ as soon as $\delta\left(\xi, \xi^{\prime}\right) \leq \exp (-((n+1) r+1))$.

Let us choose $\beta, \beta_{0}^{1 / r}<\beta<1$, and consider the space $\Gamma_{\beta}$ of functions $\phi$ on $\partial F$ such that there is a constant $C_{\beta}$ with the property that if the points $\xi$ and $\xi^{\prime}$ have the same first $n$ coordinates, then $\left|\phi(\xi)-\phi\left(\xi^{\prime}\right)\right|<C_{\beta} \beta^{n}$. For $\phi \in \Gamma_{\beta}$, denote $\|\phi\|_{\beta}$ the best constant $C_{\beta}$ in this definition. The space $\Gamma_{\beta}$ is a Banach space for the norm $\|\phi\|:=\|\phi\|_{\beta}+\max _{\partial F}|\phi|$. Proposition 4.5 says that for $p \in \mathcal{P}(B)$, the function $\Phi_{p}(\xi)=-\ln K_{\xi}\left(\xi_{1}\right)$ belongs to $\Gamma_{\beta}$.

\section{Regularity of the Martin kernel}

We want to extend the mapping $p \mapsto \Phi_{p}$ to a neighborhood $\mathcal{O}_{p}$ of $p$ in $\mathbb{C}^{B}$. Firstly, we redefine $\Gamma_{\gamma}$ as the space of complex functions $\phi$ on $\partial F$ such that there is a constant $C_{\gamma}$ with the property that, for all $n \geq 0$, if the points $\xi$ and $\xi^{\prime}$ have the same first $n$ coordinates, then $\left|\phi(\xi)-\phi\left(\xi^{\prime}\right)\right|<C_{\gamma} \gamma^{n}$. The space $\Gamma_{\gamma}$ is a complex Banach space for the norm $\|\phi\|:=\|\phi\|_{\gamma}+\max _{\partial F}|\phi|$, where $\|\phi\|_{\gamma}$ the best possible constant $C_{\gamma}$. In this section, we find a neighborhood $\mathcal{O}_{p}$ and a $\gamma=\gamma(p), 0<\gamma<1$, such that formula (6) makes sense on $\mathcal{O}_{p}$ and defines a function in $\Gamma_{\gamma}$.

In recent papers, Rugh ([Ru]) and Dubois ([Du1]) show how to extend (5) to the complex setting. In a complex Banach space $X$, they define a $\mathbb{C}$-cone as a subset invariant by multiplication by $\mathbb{C}$, different from $\{0\}$ and not containing any complex 2 -dimensional subspace in its closure. A $\mathbb{C}$-cone $\ell$ is called linearly convex if each point in the complement of $\mathcal{C}$ is contain in a complex hyperplane not intersecting $\ell$. Let $K<+\infty$. A $\mathbb{C}$-cone $\ell$ is called $K$-regular if it has some interior and if, for each vector space $P$ of complex dimension 2, there is some nonzero linear form $m \in X^{*}$ such that, for all $u \in \ell \cap P$,

$$
\|m\|\|u\| \leq K|\langle m, u\rangle|
$$

Let $\mathcal{C}$ be a linearly convex $\mathbb{C}$-cone. A projective distance $\vartheta \mathscr{C}$ on $(\mathcal{C}-\{0\}) \times(\mathcal{C}-\{0\})$ is defined as follows ([Du1], Section 2): if $f$ and $g$ are colinear, set $\vartheta_{e}(x, y)=0$; otherwise, consider the set

$$
E(f, g):=\{z, z \in \mathbb{C} \mid z f-g \notin \mathscr{C}\}
$$

and define

$$
\vartheta_{e}(f, g)=\ln \frac{b}{a}
$$

where $b=\sup |E(f, g)| \in(0,+\infty], a=\inf |E(f, g)| \in[0,+\infty)$. 
Proposition 5.1 ([Du1], Theorem 2.7). Let $X_{1}, X_{2}$ be complex Banach spaces, and let $\mathcal{C}_{1} \subset X_{1}, \mathcal{C}_{2} \subset X_{2}$ be complex cones. Let $A: X_{1} \rightarrow X_{2}$ be a linear map with $A\left(\mathcal{C}_{1}-\{0\}\right) \subset\left(\mathcal{C}_{2}-\{0\}\right)$ and assume that

$$
\Delta:=\sup _{f, g \in\left(\mathscr{C}_{1}-\{0\}\right)} \vartheta \mathscr{C}_{2}(A f, A g)<+\infty .
$$

Then, for all $f, g \in \mathcal{\ell}_{1}$,

$$
\vartheta_{\mathscr{C}_{2}}(A f, A g) \leq \tanh \left(\frac{\Delta}{4}\right) \vartheta_{\mathscr{C}_{1}}(f, g) .
$$

Proposition 5.2 ([Du1], Lemma 2.6). Let $\mathcal{C}$ be a $K$-regular, linearly convex $\mathbb{C}$-cone and let $f \sim g$ if, and only if, there is $\lambda, \lambda \neq 0$ such that $\lambda f=g$. Then $\vartheta$ e defines a complete projective metric on $\mathcal{\ell} / \sim$. Moreover, if $f, g \in \mathcal{C}$ and $\|f\|=\|g\|=1$, then there is a complex number $\rho$ of modulus $1, \rho=\rho(f, g)$, such that

$$
\|\rho f-g\| \leq K \vartheta_{e}(f, g) .
$$

Proposition 5.3 ([Ru], Corollary 5.6, [Du1], Remark 3.6). For $m \geq 1$, the set

$$
\begin{aligned}
\mathbb{C}_{+}^{m} & =\left\{u \in \mathbb{C}^{m} \mid \operatorname{Re}\left(u_{i} \overline{u_{j}}\right) \geq 0 \text { for all } i, j\right\} \\
& =\left\{u \in \mathbb{C}^{m}|| u_{i}+u_{j}|\geq| u_{i}-u_{j} \mid \text { for all } i, j\right\}
\end{aligned}
$$

is a regular linearly convex $\mathbb{C}$-cone. The inclusion

$$
\pi:\left(C_{0}-\{0\}, \vartheta\right) \rightarrow\left(\mathbb{C}_{+}^{m}-\{0\}, \vartheta_{\mathbb{C}_{+}^{m}}\right)
$$

is an isometric embedding.

Moreover, [Du1] studies and characterizes the $m \times m$ matrices which preserve $\mathbb{C}_{+}^{m}$. We need the following properties. Let $A$ be a $m \times m$ matrix with all 0 entries in $m^{\prime}$ full columns and $\lambda_{1}, \ldots, \lambda_{m}$ the $\left(m-m^{\prime}\right)$-row vectors made up of the nonzeros entries of the row vectors of $A$. Set:

$$
\delta_{k, l}:=\vartheta_{\mathbb{C}_{+}^{m-m^{\prime}}}\left(\lambda_{k}, \lambda_{l}\right), \quad \Delta_{k, l}:=\operatorname{Diam}_{\mathrm{RHP}}\left\{\frac{\left\langle\lambda_{k}, x\right\rangle}{\left\langle\lambda_{l}, x\right\rangle} \mid x \in\left(\mathbb{C}_{+}^{m-m^{\prime}}\right)^{*}, x \neq 0\right\},
$$

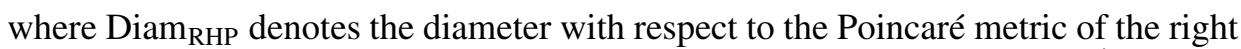
half-plane. Observe that $\operatorname{Diam}_{\vartheta_{\mathbb{C}_{+}^{m}}}\left(A\left(\mathbb{C}_{+}^{m}-\{0\}\right)\right)=\operatorname{Diam}_{\vartheta_{\mathbb{C}_{+}^{m}}}\left(A\left(\mathbb{C}_{+}^{m-m^{\prime}}-\{0\}\right)\right)$. Then we have ([Du1], Proposition 3.5):

$$
\operatorname{Diam}_{\vartheta_{\mathbb{C}_{+}^{m}}}\left(A\left(\mathbb{C}_{+}^{m}-\{0\}\right)\right) \leq \max _{k, l} \delta_{k, l}+2 \max _{k, l} \Delta_{k, l} \leq 3 \operatorname{Diam}_{\vartheta_{\mathbb{C}_{+}^{m}}}\left(A\left(\mathbb{C}_{+}^{m}-\{0\}\right)\right) .
$$

From the proof of Proposition 3.5 in [Du1], in particular from equation (3.12), it also follows that for a real matrix $A$ :

$$
\operatorname{Diam}_{\vartheta_{\mathbb{C}_{+}^{m}}}\left(A\left(\mathbb{C}_{+}^{m}-\{0\}\right)\right) \leq 3 \operatorname{Diam}_{\vartheta}\left(A\left(\mathbb{R}_{+}^{m}-\{0\}\right)\right) .
$$


Fix $p \in \mathcal{P}(B)$. We choose $\gamma=\gamma(p)<1$ such that

$$
9(\tanh )^{-1} \beta_{0}<(\tanh )^{-1}\left(\gamma^{2 r}\right) \text {. }
$$

Then for the real matrices $A=A_{1}(p), \ldots, A_{N}(p)$,

$$
\begin{aligned}
3 \operatorname{Diam}_{\vartheta_{\mathbb{C}_{+}^{m}}^{m}}\left(A\left(\mathbb{C}_{+}^{m}-\{0\}\right)\right) & \leq 9 \operatorname{Diam}_{\vartheta}\left(A\left(\mathbb{R}_{+}^{m}-\{0\}\right)\right) \\
& \leq 36(\tanh )^{-1} \beta_{0}<4(\tanh )^{-1}\left(\gamma^{2 r}\right) .
\end{aligned}
$$

Proposition 5.4. Fix $p \in \mathcal{P}(B)$. There is a neighborhood $\mathcal{O}_{p}$ of $p$ in $\mathbb{C}^{B}$ such that the mapping $p \mapsto \Phi_{p}$ extends to an analytic mapping from $\mathcal{O}_{p}$ into $\Gamma_{\gamma(p)}$.

Proof. We first extend $A_{j}, j=1, \ldots, N$, analytically on a neighborhood $\mathcal{O}_{p}$ by Proposition 4.4. Set $S=S^{2 m-1}=\left\{f \mid f \in \mathbb{C}_{+}^{m},\|f\|=1\right\}$. For each $A_{j}(q), j=$ $1, \ldots N, q \in \mathcal{O}_{p}$, and each $f \in S$ such that $A_{j}(q) f \neq 0$, we define again $T_{j}(q) f$ by

$$
T_{j}(q) f=\frac{A_{j}(q) f}{\left\|A_{j}(q) f\right\|} .
$$

For $p \in \mathcal{P}(B)$, the function $\Phi_{p}$ is given by the limit from formula (6),

$$
\Phi_{p}(\xi)=-\ln \lim _{k \rightarrow \infty} \frac{\left\langle\alpha_{1}(\xi), T_{j_{1}}(\xi) \ldots T_{j_{k-1}}(\xi) f_{0}\right\rangle}{\left\langle\alpha(\xi), T_{j_{1}}(\xi) \ldots T_{j_{k-1}}(\xi) f_{0}\right\rangle}
$$

where $f_{0} \in S$ the column vector $\{1 / \sqrt{|B|}, \ldots, 1 / \sqrt{|B|}\}$ : we use the fact that the limit of $T_{j_{1}}(\xi) \ldots T_{j_{k-1}}(\xi) f$ does not depend on the initial point $f$.

We have to show that this limit extends on some neighborhood $\mathcal{O}_{p}$ of $p$ to an analytic function into $\Gamma_{\gamma}$. Set

$$
\Phi_{p, k}(\xi):=-\ln \frac{\left\langle\alpha_{1}(\xi), A_{j_{1}}(\xi) \ldots A_{j_{k-1}}(\xi) f_{0}\right\rangle}{\left\langle\alpha(\xi), A_{j_{1}}(\xi) \ldots A_{j_{k-1}}(\xi) f_{0}\right\rangle} .
$$

We are going to find $\mathcal{O}_{p}$ and $k_{0}$ such that, for $k \geq k_{0}$, the functions $\Phi_{p, k}(\xi)$ extend to analytic functions from $\mathcal{O}_{p}$ into $\Gamma_{\gamma}$ and, as $k \rightarrow \infty$, the functions $\Phi_{p, k}(\xi)$ converge in $\Gamma_{\gamma}$ uniformly on $\mathcal{O}_{p}$. The functions $q \mapsto\left\langle\alpha_{1}(\xi), A_{j_{1}}(\xi) \ldots A_{j_{k-1}}(\xi) f_{0}\right\rangle$, $q \mapsto\left\langle\alpha(\xi), A_{j_{1}}(\xi) \ldots A_{j_{k-1}}(\xi) f_{0}\right\rangle$ are polynomials in $q$ and depend only on a finite number of coordinates of $\xi$. Therefore, if we can find a neighborhood $\mathcal{O}_{p}$ and a $k$ such that these two functions do not vanish, then $\Phi_{p, k}$ extends to an analytic function from $\mathcal{O}_{p}$ to $\Gamma_{\gamma}$.

Step 1: Contraction. By (10), (11) and Proposition 4.4, we can choose a neighborhood $\mathcal{O}_{p}$ such that for $q \in \mathcal{O}_{p}$, the diameter $\Delta$ of $A_{j}(q) \mathbb{C}_{+}^{m}$ is smaller than $4(\tanh )^{-1}\left(\gamma^{2 r}\right)$ for all $j=1, \ldots, N .^{1}$ The set $\mathscr{D}:=S \cap\left(\bigcup_{j} A_{j}(p) \mathbb{C}_{+}^{m}\right)$ is compactly contained in the interior of $S$. We choose a smaller neighborhood $\mathcal{O}_{p}$ such that if $q \in \mathcal{O}_{p}$, then

$$
\Delta<4(\tanh )^{-1}\left(\gamma^{2 r}\right) \text { and } 0 \notin A_{j}\left(\mathscr{D} \cup\left\{f_{0}\right\}\right) \text { for } j=1, \ldots, N .
$$

\footnotetext{
${ }^{1}$ One can also use directly [Du2], Theorem 4.5 .
} 
For $q \in \mathcal{O}_{p}$, the projective images $T_{j_{1}}(\xi) \ldots T_{j_{k-1}}(\xi) f_{0}$ are all defined and we have, by repeated application of (8),

$$
\vartheta_{e}\left(T_{j_{1}}(\xi) \ldots T_{j_{k-1}}(\xi) f_{0}, T_{j_{1}}(\xi) \ldots T_{j_{k-1}}(\xi) f_{k, k^{\prime}}(\xi)\right) \leq \gamma^{2(k-1) r} \vartheta\left(f_{0}, f_{k, k^{\prime}}(\xi)\right) \text {, }
$$

where $k^{\prime}>k$ and $f_{k, k^{\prime}}(\xi):=T_{j_{k}}(\xi) \ldots T_{j_{k^{\prime}-1}}(\xi) f_{0}$. The $f_{k, k^{\prime}}(\xi)$ are all in $\mathscr{D}$. Then $\vartheta_{\mathcal{C}}\left(f_{0}, f_{k, k^{\prime}}(\xi)\right) \leq C$ for all $\xi \in \partial F$, all $k, k^{\prime} \geq 1$. Set

$$
g=T_{j_{1}}(\xi) \ldots T_{j_{k-1}}(\xi) f_{0}, \quad g^{\prime}=T_{j_{1}}(\xi) \ldots T_{j_{k-1}}(\xi) f_{k, k^{\prime}}(\xi) .
$$

For all $\xi \in \partial F$, all $k, k^{\prime} \geq 1$, consider the number $\rho\left(\xi, k, k^{\prime}\right)$ associated to $g$ and $g^{\prime}$ by Proposition 5.2. We have by (9)

$$
\left|\rho\left(\xi, k, k^{\prime}\right)\right|=1 \quad \text { and } \quad\left\|\rho\left(\xi, k, k^{\prime}\right) g-g^{\prime}\right\| \leq K C \gamma^{2 k r} .
$$

Since $\alpha(p, \xi)$ and $\alpha_{1}(p, \xi)$ take finite many values, it follows that

$$
\begin{aligned}
& \left|\langle\alpha(p, \xi), g\rangle\left\langle\alpha_{1}(p, \xi), g^{\prime}\right\rangle-\left\langle\alpha(p, \xi), g^{\prime}\right\rangle\left\langle\alpha_{1}(p, \xi), g\right\rangle\right| \\
& \quad=\left|\left\langle\alpha(p, \xi), \rho\left(\xi, k, k^{\prime}\right) g\right\rangle\left\langle\alpha_{1}(p, \xi), g^{\prime}\right\rangle-\left\langle\alpha(p, \xi), g^{\prime}\right\rangle\left\langle\alpha_{1}(p, \xi), \rho\left(\xi, k, k^{\prime}\right) g\right\rangle\right| \\
& \quad \leq K C \gamma^{2 k r} .
\end{aligned}
$$

Since $g$ and $g^{\prime}$ are in the compact set $\mathscr{D} \cup\left\{f_{0}\right\}$, we can, by Proposition 3.3, choose a neighborhood $\mathcal{O}_{p}$ such that

$$
\begin{aligned}
& \mid\left\langle\alpha(\xi), T_{j_{1}}(\xi) \ldots T_{j_{k-1}}(\xi) f_{0}\right\rangle\left\langle\alpha_{1}(\xi), T_{j_{1}}(\xi) \ldots T_{j_{k^{\prime}-1}}(\xi) f_{0}\right\rangle \\
& \quad-\left\langle\alpha(\xi), T_{j_{1}}(\xi) \ldots T_{j_{k^{\prime}-1}}(\xi) f_{0}\right\rangle\left\langle\alpha_{1}(\xi), T_{j_{1}}(\xi) \ldots T_{j_{k-1}}(\xi) f_{0}\right\rangle \mid \leq K C \gamma^{2 k r}
\end{aligned}
$$

for all $q \in \mathcal{O}_{p}$, all $\xi \in \partial F$, and all $k<k^{\prime}$.

Step 2: The $\Phi_{q, k}$ extend. Recall that $D$ is the set of unit vectors in the positive quadrant. For $g, g^{\prime} \in \bigcup_{j} T_{j}(p)(D) \cup\left\{f_{0}\right\},\langle\alpha(p, \xi), g\rangle\left\langle\alpha_{1}(p, \xi), g^{\prime}\right\rangle$ is real positive and bounded away from 0 uniformly in $\xi, g$ and $g^{\prime}$. Recall the isometric inclusion $\pi: D \rightarrow S$ of Proposition 5.3. There is a neighborhood $C_{0}$ of $\pi\left(\cup_{j} T_{j}(p)(D) \cup\right.$ $\left.\left\{f_{0}\right\}\right)$ in $S$ and $\delta>0$ such that $\left|\langle\alpha(p, \xi), g\rangle\left\langle\alpha_{1}(p, \xi), g^{\prime}\right\rangle\right|>\delta$ for $g, g^{\prime} \in \mathscr{C}_{0}$. Of course, we can take $\ell_{0}$ invariant by multiplication by all $z$ with $|z|=1$. Moreover, there exists $\varepsilon>0$ such that if $\vartheta_{C_{+}^{m}}\left(g, \pi\left(\bigcup_{j} T_{j}(p)(D) \cup\left\{f_{0}\right\}\right)\right)<\varepsilon$ and $\vartheta_{C_{+}^{m}}\left(g^{\prime}, \pi\left(\bigcup_{j} T_{j}(p)(D) \cup\left\{f_{0}\right\}\right)\right)<\varepsilon$, then $\left|\langle\alpha(p, \xi), g\rangle\left\langle\alpha_{1}(p, \xi), g^{\prime}\right\rangle\right|>\delta / 2$.

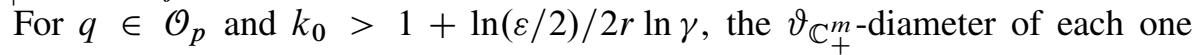
of the sets $T_{j_{1}}(q, \xi) \ldots T_{j_{k_{0}-1}}(q, \xi) S$ is smaller than $\varepsilon / 2$, for all $\xi$. As $\xi$ varies, there is only a finite number of mappings $T_{j_{1}}(q, \xi) \ldots T_{j_{k_{0}-1}}(q, \xi)$. By continuity of $q \mapsto T_{j}$ (where the $T_{j}$ s now are considered as mappings from $\ell / \sim$ into itself), there is a neighborhood $\mathcal{O}_{p}$ such that for $q \in \mathcal{O}_{p}$, the Hausdorff distance between $T_{j_{1}}(q, \xi) \ldots T_{j_{k_{0}-1}}(q, \xi) S / \sim$ and $T_{j_{1}}(p, \xi) \ldots T_{j_{k_{0}-1}}(p, \xi) S / \sim$ is smaller than $\varepsilon / 2$. It follows that if $q \in \mathcal{O}_{p}$, and $g, g^{\prime}$ are in the same $T_{j_{1}}(q, \xi) \ldots T_{j_{k_{0}-1}}(q, \xi) S$ for some $\xi$, then

$$
\left|\langle\alpha(p, \xi), g\rangle\left\langle\alpha_{1}(p, \xi), g^{\prime}\right\rangle\right|>\delta / 2 .
$$


By taking a possibly smaller $\mathcal{O}_{p}$, we have that if $q \in \mathcal{O}_{p}$, and $g, g^{\prime}$ are in the same $T_{j_{1}}(q, \xi) \ldots T_{j_{k_{0}-1}}(q, \xi) S$ for some $\xi$, then

$$
\left|\langle\alpha(q, \xi), g\rangle\left\langle\alpha_{1}(q, \xi), g^{\prime}\right\rangle\right|>\delta / 4
$$

In particular this last expression does not vanish and $\Phi_{q, k}$ is an analytic function on $\mathcal{O}_{p}$ for $k \geq k_{0}$.

Step 3: The $\Phi_{q, k}$ converge uniformly on $\partial F$. Take a neighborhood $\mathcal{O}_{p}$ and $k_{0}$ such that for $q \in \mathcal{O}_{p}$ the conclusions of steps 1 and 2 hold. We claim that for all $\varepsilon>0$, there is $k_{1}$ such that for $k, k^{\prime} \geq k_{1}, q \in \mathcal{O}_{p}, \max _{\xi}\left|\Phi_{q, k}(\xi)-\Phi_{q, k^{\prime}}(\xi)\right|<\varepsilon$. Suppose that $k_{1}>k_{0}$. We have to estimate

$$
\max _{\xi}\left|\ln \frac{\left\langle\alpha(\xi), T_{j_{1}}(\xi) \ldots T_{j_{k-1}}(\xi) f_{0}\right\rangle}{\left\langle\alpha(\xi), T_{j_{1}}(\xi) \ldots T_{j_{k^{\prime}-1}}(\xi) f_{0}\right\rangle} \frac{\left\langle\alpha_{1}(\xi), T_{j_{1}}(\xi) \ldots T_{j_{k^{\prime}-1}}(\xi) f_{0}\right\rangle}{\left\langle\alpha_{1}(\xi), T_{j_{1}}(\xi) \ldots T_{j_{k-1}}(\xi) f_{0}\right\rangle}\right| .
$$

By the conclusions of steps 1 and 2, this quantity is smaller that $C \max \left\{\gamma^{2 k r}, \gamma^{2 k^{\prime} r}\right\}$. This is smaller than $\varepsilon$ if $k_{1}$ is large enough.

Step 4: The $\Phi_{q, k}$ converge in norm $\|\cdot\|_{\gamma(p)}$. With the same $\mathcal{O}_{p}, k_{0}$, we now claim that for all $\varepsilon>0$, there is $k_{2}=\max \left\{k_{0}, \ln \gamma / r \ln \varepsilon\right\}$ such that for $k, k^{\prime} \geq k_{2}$ and $q \in \mathcal{O}_{p},\left\|\Phi_{q, k}(\xi)-\Phi_{q, k^{\prime}}(\xi)\right\|_{\gamma}<\varepsilon$. Let $\xi$, $\xi^{\prime}$ be two points of $\partial F$ with $\delta\left(\xi, \xi^{\prime}\right) \leq \exp (-((n+1) r+1))$. We want to show that there is a constant $C$ independent on $n$, such that

$$
\left|\Phi_{q, k}(\xi)-\Phi_{q, k^{\prime}}(\xi)-\Phi_{q, k}\left(\xi^{\prime}\right)+\Phi_{q, k^{\prime}}\left(\xi^{\prime}\right)\right| \leq C \gamma^{(n+1) r+1} \varepsilon
$$

for all $q \in \mathcal{O}_{p}$, all $k, k^{\prime} \geq k_{2}$. Since $k, k^{\prime} \geq k_{0}$, the difference $\Phi_{q, k}(\xi)-\Phi_{q, k^{\prime}}(\xi)$ is given by

$$
\Phi_{q, k}(\xi)-\Phi_{q, k^{\prime}}(\xi)=\ln \frac{\left\langle\alpha_{1}, T_{j_{1}} \ldots T_{j_{k^{\prime}-1}} f_{0}\right\rangle}{\left\langle\alpha_{1}, T_{j_{1}} \ldots T_{j_{k-1}} f_{0}\right\rangle} \frac{\left\langle\alpha, T_{j_{1}} \ldots T_{j_{k-1}} f_{0}\right\rangle}{\left\langle\alpha, T_{j_{1}} \ldots T_{j_{k^{\prime}-1}} f_{0}\right\rangle} .
$$

For $k, k^{\prime} \leq n+1, \Phi_{q, k}(\xi)-\Phi_{q, k^{\prime}}(\xi)=\Phi_{q, k}\left(\xi^{\prime}\right)-\Phi_{q, k^{\prime}}\left(\xi^{\prime}\right)$, and there is nothing to prove.

Assume that $k^{\prime}>k \geq n+1$. Step 3 shows that both $\left|\Phi_{q, k}(\xi)-\Phi_{q, k^{\prime}}(\xi)\right|$ and $\left|\Phi_{q, k}\left(\xi^{\prime}\right)-\Phi_{q, k^{\prime}}\left(\xi^{\prime}\right)\right|$ are smaller than $C \gamma^{2 k r} \leq C \gamma^{n r} \gamma^{k r} \leq C \gamma^{n r} \varepsilon$.

The remaining case, when $k_{0} \leq k \leq n+1 \leq k^{\prime}$, clearly follows from the other two, and this shows step 4.

Finally we have that the functions $\Phi_{p, k}$ are analytic and converge uniformly in $\Gamma_{\gamma}$ on a neighborhood $\mathcal{O}_{p}$ of $p$. The limit is an analytic function on $\mathcal{O}_{p}$.

\section{Proof of Theorem 1.1}

In this section, we consider $\partial F$ as a subshift of finite type and let $\tau$ be the shift transformation on $\partial F$ :

$$
\tau \xi=\eta_{1} \eta_{2} \ldots \quad \text { with } \eta_{n}=\xi_{n+1} .
$$


For $\gamma<1$ and $\phi \in \Gamma_{\gamma}$ with real values, we define the transfer operator $\mathscr{L}_{\phi}$ on $\Gamma_{\gamma}$ by

$$
\mathscr{L}_{\phi} \psi(\xi):=\sum_{\eta \in \tau^{-1} \xi} e^{\phi(\eta)} \psi(\eta)
$$

Then $\mathscr{L}_{\phi}$ is a bounded operator in $\Gamma_{\gamma}$ and, by Ruelle's transfer operator theorem (see e.g. [Bo]), there exists a number $P(\phi)$, a positive function $h_{\phi} \in \Gamma_{\gamma}$ and an unique linear functional $v_{\phi}$ on $\Gamma_{\gamma}$ such that

$$
\mathscr{L}_{\phi} h_{\phi}=e^{P(\phi)} h_{\phi}, \quad \mathscr{L}_{\phi}^{*} v_{\phi}=e^{P(\phi)} v_{\phi} \quad \text { and } \quad v_{\phi}(1)=1 .
$$

The functional $v_{\phi}$ extends to probability measure on $\partial F$ and is the only eigenvector of $\mathscr{L}_{\phi}^{*}$ with that property. Moreover, $\phi \mapsto \mathscr{L}_{\phi}$ is a real analytic map from $\Gamma_{\gamma}$ to the space of linear operators on $\Gamma_{\gamma}$ ([R1], p. 91). Consequently, the mapping $\phi \mapsto v_{\phi}$ is real analytic from $\Gamma_{\gamma}$ into the dual space $\Gamma_{\gamma}^{*}$ (see e.g. [Co], Corollary 4.6). By Proposition 5.4, the mapping $p \mapsto v_{\Phi_{p}}$ is real analytic from a neighborhood of $p$ in $\mathcal{P}(B)$ into the space $\Gamma_{\gamma(p)}^{*}$.

The main observation is that $\mathscr{L}_{\Phi_{p}}^{*} p^{\infty}=p^{\infty}$ for all $p \in \mathcal{P}(B)$; this implies that $P\left(\Phi_{p}\right)=0$ and that the distribution $v_{\Phi_{p}}$ is the restriction of the measure $p^{\infty}$ to any $\Gamma_{\gamma}$ such that $\Phi_{p} \in \Gamma_{\gamma}$. Indeed, we have

$$
\frac{d \tau_{*} p^{\infty}}{d p^{\infty}}(\xi)=\frac{d\left(\xi_{1}\right)_{*} p^{\infty}}{d p^{\infty}}=K_{\xi}\left(\xi_{1}\right)=e^{\Phi_{p}(\xi)}
$$

so that, for all continuous $\psi$,

$$
\int\left(\mathscr{L}_{\Phi_{p}} \psi\right) d p^{\infty}=\sum_{a} \int_{a \xi, \xi_{1} \neq a^{-1}} \frac{d p^{\infty}(a \xi)}{d p^{\infty}(\xi)} \psi(a \xi) d p^{\infty}(\xi)=\int \psi d p^{\infty} .
$$

Recall the equations (1) and (2) for $h_{p}$ and $\ell_{p}$. The linear drift $\ell_{p}$ is given by a finite sum (in $x$ ) of integrals with respect to $p^{\infty}$ of the functions $\xi \mapsto \theta_{\xi}(x)$. Since these functions only depend on a finite number of coordinates in $\partial F$, they belong to $\Gamma_{\gamma}$ for all $\gamma<1$. Since $p \mapsto v_{\Phi_{p}}$ is real analytic from a neighborhood of $p$ into $\Gamma_{\gamma(p)}^{*}$, $p \mapsto \ell_{p}$ is real analytic on a neighborhood of $p$. Since this is true for all $p \in \mathcal{P}(B)$, the function $p \mapsto \ell_{p}$ is real analytic on $\mathcal{P}(B)$.

The argument is the same for $h_{p}$, since the function $\ln \frac{d x_{*}^{-1} p^{\infty}}{d p^{\infty}}(\xi)=\ln K_{\xi}\left(x^{-1}\right) \in$ $\Gamma_{\gamma}$ for all $x$ and for all $\gamma, \beta<\gamma<1$ and the mappings $p \mapsto \ln K_{\xi}\left(x^{-1}\right)$ are real analytic from a neighborhood of $p$ into $\Gamma_{\gamma}(p)$. Indeed, $\ln K_{\xi}\left(\xi_{1}\right) \in \Gamma_{\beta}$ by Proposition 4.5 and $p \mapsto \ln K_{\xi}\left(\xi_{1}\right)$ is real analytic into $\Gamma_{\gamma(p)}$ by Proposition 5.4. Moreover, if $a$ is a generator different from $\xi_{1}$, then $\ln K_{\xi}(a)=-\ln K_{a^{-1} \xi}\left(a^{-1}\right)$ also lies in $\Gamma_{\beta}$ and $p \mapsto \ln K_{\xi}(a)$ is real analytic into $\Gamma_{\gamma(p)}$ as well. For a general $x \in F$, $x=a_{1} \ldots a_{t}$, write

$$
K_{\xi}\left(x^{-1}\right)=K_{\xi}\left(a_{t}^{-1} \ldots a_{1}^{-1}\right)=K_{\xi}\left(a_{t}^{-1}\right) K_{a_{t} \xi}\left(a_{t-1}^{-1}\right) \ldots K_{a_{2} \ldots a_{t} \xi}\left(a_{1}^{-1}\right) .
$$


This completes the proof of Theorem 1.1. For the proof of Theorem 1.2, fix an origin $o \in \mathbb{H}^{k}$. Then $\pi(F) o$ accumulates to the boundary of $\mathbb{H}^{k}$ in a Cantor set $\Lambda$ called the limit set of $\pi(F)$. The mapping $\pi_{o}: F \rightarrow \mathbb{H}^{n}, \pi_{o}(x)=x \cdot o$, extends to a Hölder continuous mapping $\pi_{o}$ from $\partial F$ to the limit set $\Lambda$ of $\pi(F)$. We can express the exponent $\gamma_{p}$ as

$$
\gamma_{p}=\lim _{n} \frac{1}{2 n} \sum_{x \in F} d\left(o, \pi_{o}(x)\right) p^{(n)}(x)
$$

where the distance $d$ is the hyperbolic distance in $\mathbb{H}^{k}$. We obtain, in the same way as for formula (2),

$$
\begin{aligned}
\gamma_{p} & =\frac{1}{2} \sum_{x \in F}\left(\int_{\Lambda} \Theta_{\zeta}\left(\pi_{o}\left(x^{-1}\right)\right) d\left(\left(\pi_{o}\right)_{*} p^{\infty}\right)(\zeta)\right) p(x) \\
& =\frac{1}{2} \sum_{x \in F}\left(\int_{\partial F} \Theta_{\pi_{o}(\xi)}\left(\pi_{o}\left(x^{-1}\right)\right) d\left(p^{\infty}\right)(\xi)\right) p(x),
\end{aligned}
$$

where $\Theta_{\zeta}$ is now the Busemann function of $\mathbb{H}^{k}: \Theta_{\zeta}(z):=\lim _{w \rightarrow \zeta} d(w, z)-d(w, o)$. Since, for all $x \in F$, the function $\xi \mapsto \Theta_{\pi_{o}(\xi)}\left(\pi_{o}(x)\right)$ is a $\rho$-Hölder continuous function for some fixed $\rho$, we deduce as above that $p \mapsto \gamma_{p}$ is real analytic on $\mathcal{P}(B)$.

Note added in proof. Analyticity of the entropy in related circumstances is also obtained in [G2] and [HMP].

\section{References}

[Bi] G. Birkhoff, Extensions of Jentzsch's theorem. Trans. Amer. Math. Soc. 85 (1957), 219-227. Zbl 0079.13502 MR 0087058

[Bo] R. Bowen, Equilibrium states and the ergodic theory of Anosov diffeomorphisms. 2nd revised ed., Lecture Notes in Math. 470, Springer-Verlag, Berlin 2008. Zbl 1172.37001 MR 2423393

[Co] G. Contreras, Regularity of topological and metric entropy of hyperbolic flows. Math. Z. 210 (1992), 97-111. Zbl 0735.58026 MR 1161172

[De1] Y. Derriennic, Marche aléatoire sur le groupe libre et frontière de Martin. Z. Wahrscheinlichkeitstheorie und Verw. Gebiete 32 (1975), 261-276. Zbl 0364.60117 MR 0388545

[De2] Y. Derriennic, Quelques applications du théorème ergodique sous-additif. In Conference on random walks (Kleebach, 1979), Astérisque 74, Soc. Math. France, Paris 1980, 183 201. Zbl 0446.60059 MR 588163

[DG] Y. Derriennic and Y. Guivarc'h, Théorème de renouvellement pour les groupes non moyennables. C. R. Acad. Sci. Paris Sér. A 277 (1973), 613-615. Zbl 0272.60005 MR 0328990 
[Du1] L. Dubois, Projective metrics and contraction principles for complex cones. J. Lond. Math. Soc. (2) 79 (2009), 719-737. Zbl 1172.15011 MR 2506695

[Du2] L. Dubois, An explicit Berry-Esséen bound for uniformly expanding maps on the interval. Israel J. Math. 186 (2011), 221-250.

[DM] E. B. Dynkin and M. B. Maljutov, Random walk on groups with a finite number of generators. Dokl. Akad. Nauk SSSR 137 (1961), 1042-1045; English transl. Soviet Math. Dokl. 2 (1961), 399-402. Zbl 0214.44101 MR 0131904

[Er] A. Erschler, On continuity of range, entropy and drift for random walks on groups. In Random walks, boundaries and spectra. Progr. Probab. 64, Birkhäuser, Basel 2011, 55-64. Zbl 1225.60012

[EK] A. Erschler and V. A. Kaimanovich, Continuity of entropy for random walks on hyperbolic groups. In preparation.

[Fu] H. Furstenberg, Random walks and discrete subgroups of Lie groups. In Advances in probability and related topics, Vol. 1, Dekker, New York 1971, 1-63. Zbl 0221.22008 MR 0284569

[G1] L. A. Gilch, Rate of escape of random walks on free products. J. Aust. Math. Soc. 83 (2007), 31-54. Zbl 1147.60030 MR 2378433

[G2] L. A. Gilch, Asymptotic entropy of random walks on free products. Electron. J. Probab. 16 (2011), 76-105. Zbl 1225.60126 MR 2749773

[HMP] G. Han, B. Marcus, and Y. Peres, A note on a complex Hilbert metric with application to domain of analyticity for entropy rate of hidden Markov processes. In Entropy of hidden Markov processes and connections to dynamical systems, London Math. Soc. Lecture Note Ser. 385, Cambridge University Press, Cambridge 2011, 98-116. arXiv:0905.2479v1 [math.DS]

[H] H. Hennion, Dérivabilité du plus grand exposant caractéristique des produits de matrices aléatoires indépendantes à coefficients positifs. Ann. Inst. H. Poincaré Probab. Statist. 27 (1991), 27-59. Zbl 0724.60009 MR 1098563

[Ka] V. A. Kaimanovich, The Poisson formula for groups with hyperbolic properties. Ann. of Math. (2) 152 (2000), 659-692. Zbl 0984.60088 MR 1815698

[KL] A. Karlsson and F. Ledrappier, Linear drift and Poisson boundary for random walks. Pure Appl. Math. Q. 3 (2007), 1027-1036. Zbl 1142.60035 MR 2402595

[Le] F. Ledrappier, Some asymptotic properties of random walks on free groups. In Topics in probability and Lie groups: boundary theory, CRM Proc. Lecture Notes 28, Amer. Math. Soc., Providence, RI, 2001, 117-152. Zbl 0994.60073 MR 1832436

[M] J. Mairesse, Randomly growing braid on three strands and the manta ray. Ann. Appl. Probab. 17 (2007), 502-536. Zbl 1146.60009 MR 2308334

[MM] J. Mairesse and F. Mathéus, Random walks on free products of cyclic groups. J. Lond. Math. Soc. (2) 75 (2007), 47-66. Zbl 1132.60054 MR 2302729

[P] Y. Peres, Domains of analytic continuation for the top Lyapunov exponent. Ann. Inst. H. Poincaré Probab. Statist. 28 (1992), 131-148. Zbl 0794.58023 MR 1158741

[R1] D. Ruelle, Thermodynamic formalism. Encyclopedia Math. Appl. 5, Addison-Wesley Publishing Co., Reading, Mass. 1978. Zbl 0401.28016 MR 511655 
[R2] D. Ruelle, Analycity properties of the characteristic exponents of random matrix products. Adv. Math. 32 (1979), 68-80. Zbl 0426.58018 MR 534172

[R3] D. Ruelle, Repellers for real analytic maps. Ergodic Theory Dynam. Systems 2 (1982), 99-107. Zbl 0506.58024 MR 684247

[Ru] H. H. Rugh, Cones and gauges in complex spaces: spectral gaps and complex Perron-Frobenius theory. Ann. of Math. (2) 171 (2010), 1707-1752. Zbl 1208.15026 MR 2680397

Received September 27, 2010; revised December 12, 2010

F. Ledrappier, LPMA, UMR CNRS 7599, Université Paris 6, Boîte Courrier 188,

4, Place Jussieu, 75252 Paris cedex 05, France

E-mail: francois.ledrappier@upmc.fr 